\title{
Posverdad y noticias falsas: esquemas perversos de interferencia democrática
} Post-truth and Fake News: Perverse Schemes of Democratic Interference

\author{
Manuel Bermúdez VÁzQuez \\ Universidad de Córdoba \\ manuel.bermudez@uco.es
}

Resumen: La dinámica de nuestro tiempo nos ha llevado a una situación especialmente delicada para nuestros sistemas políticos. En el análisis que aquí presentamos damos cuenta de cuatro mecanismos que, aunados, pueden suponer una seria amenaza para el funcionamiento de la democracia. Estos mecanismos son: la demagogia, las noticias falsas, la posverdad y la carencia de pensamiento crítico. Con una presentación breve pero contundente de los efectos devastadores que estos elementos están ya provocando lo que nos proponemos es poner sobre aviso a la sociedad para tratar de aplicar las medidas correctoras necesarias.

Palabras clave: posverdad, noticias falsas, pensamiento crítico, filosofía política. 


\begin{abstract}
Our time have led us to a particularly complex situation. We present here an analysis in which four political and teaching mechanisms pose a very serious threat to the functioning of our democracy. These mechanisms are: demagogy, fake news, posttruth and the lack of critical thinking. With a brief but forceful presentation of the devastating effects that these elements are already causing, what we propose is to alert society in order to allow the necessary corrective measures.
\end{abstract}

Keywords: post-truth, fake news, critical thinking, political theory.

Fecha de recepción: $27 / 7 / 2020$

Fecha de aceptación: 17/2/2021 


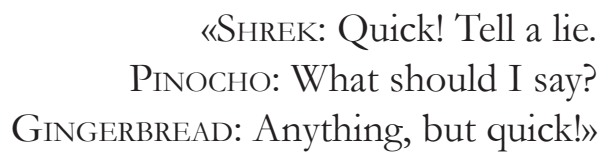

Escena de Shrek 2 (2004)

«Lo que el público quiere es la imagen de la pasión, no la pasión en sí. No hay en la lucha problema con la verdad como también ocurre en el teatro»

Roland Barthes, Mitologías (1957)

\section{Introducción}

En 1957 el semiólogo y crítico literario francés Roland Barthes publicó un libro titulado Mitologías. Este libro es una amena colección de ensayos y el primero de ellos se titula «El mundo de la lucha libre». No deja de ser sorprendente que en una obra de marcado carácter intelectual y de obvia profundidad filosófica se introduzca una cuestión tan aparentemente vulgar como el ámbito de la lucha libre. Sin embargo, la aguda visión analítica de Barthes en ese texto de pocas páginas nos permite iniciar estas líneas reconociendo su perspicacia. En este primer ensayo de su libro, el autor francés hace una descripción de la lucha libre como una suma de espectáculos en la que nadie se cree lo que está ocurriendo en la lona. Primero compara la lucha libre con el judo. En este nadie finge, la pugna se produce con gestos medidos, sin grandilocuencias, poniendo el máximo esfuerzo en el enfrentamiento. Sin embargo, en la lucha libre todo es exa- 
geración, fingimiento, sobreactuación. Para Roland Barthes no hay diferencia entre la lucha libre y el teatro y ello no es ningún desdoro para la primera (Barthes, 1999). A pesar de esta actuación premeditada y sin ocultación, disimulada, la lucha libre tiene cientos de miles de seguidores por todo el mundo. Pues esta imagen me sirve como analogía para presentar el fenómeno de la posverdad y la amenaza que implica.

En el año 2016 el diccionario de Oxford decretó que la palabra del año en inglés era el adjetivo post-truth. Esta palabra se refería a aquellas circunstancias en las que los hechos objetivos tenían menos importancia a la hora de influir en la opinión pública que las apelaciones a la emoción y a las creencias personales (Oxford, 2016). Según las declaraciones del propio diccionario de Oxford, el concepto de post-truth llevaba existiendo al menos desde la década anterior, pero el aumento sustancial de la frecuencia de uso de la palabra llevó a considerarla como la candidata idónea para ser nombrada palabra del año en inglés ${ }^{1}$. Sobre todo, se vinculó a los contextos de las elecciones presidenciales de 2016 en Estados Unidos y el referéndum del mismo año en el Reino Unido sobre la permanencia en la Unión Europea. Entre los argumentos esgrimidos por la comisión que terminó declarándola palabra del año se encontraba el hecho de que post-truth había pasado de ser una palabra poco utilizada, más bien situada en la periferia metafórica del lenguaje, a aparecer con mucha fre-

\footnotetext{
1 Sabemos que en esto hay una imprecisión. La palabra post-truth tiene su origen en 1992 en el artículo de Steve Tesich, «A government of lies», escrito en mitad del escándalo del Irán-Contra y la Guerra del Golfo. Probablemente las autoridades del diccionario de Oxford solo conocían la obra de Ralph Keyes del año 2004, titulada The Post-Truth Era: Dishonesty and Deception in Contemporary Life, donde se presenta un análisis somero sobre el uso de la mentira en la sociedad contemporánea (Bermúdez, 2019: 342). La bibliografía sobre el fenómeno de la posverdad es cada vez mayor. La red está repleta de blogs, artículos y ensayos al respecto. Si embargo, desde la perspectiva académica, el artículo en español más abarcador y que realmente aporta un auténtico estado de la cuestión sobre el tema que hemos encontrado es el del catedrático de la UGR Juan Antonio Nicolás (Nicolás, 2019).
}

Bermúdez Vázquez, Manuel (2021). «Posverdad y noticias falsas: esquemas perversos de interferencia democrática». Monograma. Revista Iberoamericana de Culturay Pensamiento, n. ${ }^{\circ}$ 8, pp. 17-38. doi: 10.36008/monograma.2021.08.1422. http: revistamonograma.com. ISSN: 2603-5839. 
cuencia no solo en redes sociales, sino también en publicaciones de nivel y ello sin necesidad de aclarar o definir el término, lo cual demostraba que ya era reconocido por todo el mundo. El término había pasado de ser relativamente nuevo a ser ampliamente empleado y comprendido por mucha gente. Todo ello en un periodo de tiempo bastante breve, aproximadamente un año. Esta fue otras de las cuestiones que pusieron en claro el impacto a todos los niveles que tuvo esta palabra. Al ser un adjetivo en inglés, la palabra por sí sola no podía emplearse con comodidad, de modo que la expresión con la que aparecía más vinculada era el concepto «post-truth politics», en español sería algo así como «políticas posverdaderas». Esta traducción ya pone de manifiesto la dificultad que tenemos en español para respetar el sentido del adjetivo post-truth. De hecho, el término ha sido traducido como «posverdad», que es un sustantivo, de ahí que, por mor de la acribia terminológica, quizá sería más preciso y más oportuno hablar más bien de «mecanismos posverdaderos» o «recursos posverdaderos» más que de «posverdad» (como ya hemos indicado en otra parte: Bermúdez, 2019: 342-343).

Sea como fuere, el hecho de que esta palabra alcanzara la relevancia suficiente como para convertirse en el término más influyente del año 2016 ya señala una dirección: la insidiosa inoculación de mecanismos espurios en el funcionamiento de las democracias contemporáneas. Además, los mecanismos posverdaderos pusieron de manifiesto la enorme fuerza de la propaganda cuando se apoya sobre prejuicios o expectativas ya existentes ( $\mathrm{Ta}$ rín, 2018). Por otro lado, con el advenimiento de los mecanismos posverdaderos, hemos asistido a la defunción del discurso de logos y al afianzamiento del discurso de pathos. Dicho con otras palabras, las apelaciones emotivas han enterrado a los razonamientos argumentados y ordenados. Veamos con algún detalle. 
Aristóteles, en su obra Retórica, presentó con una aguda visión analítica las distintas partes que puede presentar un discurso. Estas eran: ethos, logos y pathos. El ethos tenía como meta fundamental que el auditorio se identificara con el orador y confiara en él. El logos consistía en los razonamientos y argumentos que se empleaban para defender una postura o presentar una posición. El pathos estaría formado por las apelaciones emotivas dirigidas a conmover al auditorio y hacerlo sentir en sintonía con el orador (Aristóteles, 1994; Leith, 2011). Pues bien, con la llegada de los mecanismos posverdaderos hemos podido constatar que el logos ha desaparecido prácticamente del discurso político y todo el espacio ha quedado ocupado, en su mayor medida, por apelaciones emotivas que forman parte del pathos aristotélico. Si el ethos no ha desaparecido es debido a que los oradores necesitan una base sobre la que apoyar su mensaje (Bermúdez, 2019: 349-351; Bermúdez y Casares, 2019: 64-65).

Así pues, en este panorama podemos constatar que se producen una serie de mecanismos perversos que interfieren en el normal desarrollo democrático y que son un riesgo tanto a corto como a largo plazo para el sostenimiento de nuestras sociedades. A fin de cuentas, en el actual sistema, en parte heredero de la tradición liberal, se supone que los ciudadanos deben poder tomar sus decisiones políticas estando bien informados (Rodríguez, 2008: 13-15). Los partidos políticos deben presentar sus propuestas con veracidad y honestidad y los ciudadanos, partiendo de esta información confiable y contrastable, podrían elegir la opción que estimen oportuno. Pues bien, si hemos permitido que los mecanismos posverdaderos se inmiscuyan en este proceso y lo contaminen, estaríamos hablando de una injerencia irreparable que afectaría a los mimbres básicos de la democracia (Bermúdez y Casares, 2019). 
«La gente no quiere estar informada. La gente quiere sentirse informada» Roger Ailes, The Loudest Voice

\section{Análisis}

En el título el presente artículo hablamos de «esquemas perversos». Desde nuestro análisis, hemos vertebrado en torno a cuatro factores estos elementos que consideramos espurios dentro del sistema democrático: la demagogia, el aumento de las noticias falsas, el auge de los mecanismos posverdaderos y la ausencia de contenidos vinculados al pensamiento crítico en nuestros sistemas educativos.

\section{Demagogia}

La palabra demagogia, de tan usada, de tan manida, parece prostituida, como si su sentido pudiera hacerse flexible para indicar cualquier cosa negativa que el orador quiera hacer recaer sobre un rival o un enemigo. Ante esta situación, uno de los métodos de la filosofía para precisar los conceptos consiste en ir a su origen, en este caso a su origen etimológico. La palabra demagogia es un término procedente del griego clásico que tiene dos elementos. La primera parte es el sustantivo «demos», que significa, para lo que aquí nos atañe, «pueblo». La segunda parte es un verbo, «ago», que significa «conducir». De modo que a través de la palabra demagogia lo que estamos tratando de transmitir es la idea de conducir al pueblo. Su origen tiene una raíz peyorativa porque ya desde el nacimiento de la democracia en la antigua Grecia se vio que el principal enemigo de la misma era la demagogia. De hecho, Aristóteles en el libro III de su Politica considera sin ambages que la desviación de la democracia sería 
la demagogia (Aristóteles, 1988: 171-173). El demagogo actuaría como el pastor que pretende llevar al rebaño al redil que más le conviene a él. Para ello, y abusando de la metáfora, utilizaría dos elementos, el cayado, por una parte y el perro ovejero que le ayuda en el manejo del rebaño, por otra. Estos dos elementos podrían ser, en la vida política real y prescindiendo ya de la metáfora, el populismo. No deja de resultar curioso que el mismo año que el diccionario de Oxford declaraba post-truth la palabra del año, la Fundéu, Fundación del Español Urgente, vinculada a la Real Academia Española, considerara que la palabra del año 2016 en español era «populismo» (Fundéu, 2016). Entre las razones argüidas por la Fundéu para otorgar esa mención de palabra del año al término "populismo» se encuentra el hecho de que se ha convertido en un término muy extendido, empleado en el discurso político ampliamente y, al mismo tiempo, también ha sido una palabra que ha sufrido un proceso paulatino de cambio de significación convirtiéndose, finalmente, en un término fuertemente peyorativo (Fundéu, 2016). Tras ese cambio de significado, populismo ha pasado a referirse a movimientos de cualquier ideología que persiguen la apelación emotiva dirigida al ciudadano y la propuesta de soluciones simples a problemas realmente complejos que, en realidad, no pueden resolverse como se trata de hacer creer. Recuérdese que Aristóteles llamaba «pathos» a las apelaciones emotivas recogidas en un discurso político. De este modo podemos ver una conexión entre el discurso populista y el pathos aristotélico. Sea como fuere, la clave fundamental de este primer factor tenido en consideración es que la demagogia y el populismo ponen en riesgo el sostenimiento de nuestro sistema democrático, al menos tal y como fue concebido. No obstante, los mecanismos reguladores y de mantenimiento del equilibrio que la propia democracia posee podrían ser suficientes para afrontar esta amenaza si fuera el caso de que solo se estuviera 
dando este fenómeno. Sin embargo, la demagogia no viene sola, forma parte, como hemos tratado de indicar en las líneas anteriores, de un mecanismo perverso que tiene otros tres elementos en perfecta sintonía, un auténtico contubernio. Pasemos a ver el segundo de ellos.

\section{Noticias falsas}

El aumento de las noticias falsas está alcanzando tal calibre que ya aparece para todos los organismos democráticos como una auténtica amenaza. La propia Comisión Europea ha mostrado su preocupación e interés por dotar de herramientas a la ciudadanía para afrontar el desafío que suponen las noticias falsas (Comisión Europea, 2019; Comisión Europea, 2020). Existen estudios muy serios que consideran que para el año 2022, el $50 \%$ de las noticias que circulen por la red serán noticias falsas y otros que predicen que para ese año los ciudadanos de las economías desarrolladas consumirán más noticias falsas que verdaderas (Gartner, 2017; Martínez, 2019). Este dato podría no ser muy relevante si la ciudadanía tuviera fuentes de información fiables y variadas, pero la auténtica dimensión de la amenaza que viene aparejada con el aumento de las noticias falsas se puede contemplar cuando cruzamos el dato anterior con este: el 60\% de la población española solo se informa a través de internet y las redes sociales (otros países tendrían porcentajes similares: 66\% Portugal, 56\% Dinamarca, 68\% Estados Unidos) (Eurostat, 2020; Shearer y Matsa, 2018). Además, este porcentaje se dispara entre la población de menor edad (Eurostat, 2020). Esta situación arroja un análisis simple. Un porcentaje elevado de la población va a obtener su información de fuentes envenenadas repletas de noticias falsas. No es nuestro interés ocuparnos aquí de las motivaciones que puede haber tras estas noticias 
falsas: injerencias políticas, presiones de potencias extranjeras, intereses comerciales, maniobras de marketing, etc. Sean estas cuales sean, la realidad es que convierte en espurias las fuentes de las que dimanan estas noticias falsas. Así, asistimos con preocupación a la desinformación interesada de gran parte de la población que luego tomará decisiones de todo tipo (políticas, económicas, de consumo, medioambientales, etc.) basándose en noticias falsas que pueden conducirnos al error y a la alienación.

Las noticias falsas no son algo nuevo. De hecho, son tan antiguas como la humanidad, al igual que la propaganda. Desde el albor de los tiempos en el que algún líder tribal quisiera aumentar su dominación y control sobre el resto, probablemente se usaron herramientas similares a las que hoy en día definimos como noticias falsas o mecanismos propagandísticos. Ahora bien, el origen de las noticias falsas de nuestro tiempo, apoyadas en el desarrollo tecnológico y el auge de internet y las redes sociales, se puede localizar en los primeros pasos, titubeantes, de las noticias en la red hace veinte o veinticinco años. El objetivo inicial era atraer la atención del auditorio y lograr que clicara sobre el titular que se proponía. De ahí surgieron los llamados «ciberanzuelos», la manera que hemos tenido en español de traducir la palabra clickbait (Fundéu, 2017). Los titulares más sensacionalistas, las expresiones más llamativas tenían por meta atraer la atención de los lectores para aumentar el número de accesos a la noticia o a la web $\mathrm{y}$, de este modo, hacer crecer los beneficios, pues las grandes compañías de publicidad pagan por el número de personas que acceden a sus anuncios. La clave de las noticias falsas es que son falsas deliberadamente. Se construye la noticia falsa con algún propósito espurio y se pretende engañar al auditorio (McIntyre, 2020: 120). Esta es la cuestión que las hace tan perniciosas para el ámbito político. Llenar las redes sociales con noticias falsas sobre cuestiones de todo tipo desorienta a la ciudadanía y la 
lleva a tomar decisiones que no se corresponden con la realidad ni encajan con sus intereses. Estaríamos asistiendo a un engaño de proporciones astronómicas. Una maniobra de manipulación perfecta que, además, es recibida por una audiencia especialmente debilitada para hacerle frente. Pasemos a analizar someramente el tercer elemento de este mecanismo perverso.

\section{Posverdad}

El Diccionario de la Real Academia recoge en su actualización de 2020 este término como un sustantivo con la siguiente definición: «Distorsión deliberada de una realidad, que manipula creencias y emociones con el fin de influir en la opinión pública y en actitudes sociales» (RAE, 2020). Ya hemos hablado en la introducción del auge de esta palabra y cómo parte de la población la usa y comprende su sentido. La posverdad plantea una serie de mecanismos perniciosos a través de los cuales un ciudadano cualquiera está dispuesto a creer que algo es verdad porque encaja con sus sentimientos y prejuicios antes que con la realidad. Esto, de por sí, ya pone de manifiesto la pérdida de influencia en el discurso público del razonamiento argumentado, los hechos o los datos empíricos frente a la nueva dictadura de la emoción. Parece como si Óscar Wilde hubiese tenido una intuición formidable cuando sentenció burlonamente: «Los argumentos ya no convencen a nadie».

Desde mi punto de vista, tanto el auge de las noticias falsas como el fenómeno de la posverdad son eventos entrelazados. Tanto el primero como el segundo beben directamente de una pérdida paulatina de la credibilidad de los grandes medios de comunicación tradicionales. Por ejemplo, tras la victoria de Donald Trump en las elecciones presidenciales de Estados Unidos en 2016, hubo tres grandes medios de comunicación que hi- 
cieron un seguimiento exhaustivo de las declaraciones del presidente. Estos medios fueron el New York Times, el Washington Post y la revista Time. Las tres publicaciones dedicaron tiempo, esfuerzo y recursos para recoger las declaraciones del presidente estadounidense y se convirtieron en los principales fiscalizadores de este. Para el mes de julio de 2020, el Washington Post ya había localizado más de 20.000 mentiras, afirmaciones engañosas y directamente embustes sin fundamento dichas por Donald Trump (Washington Post, 2020; The Guardian, 2020). En una democracia saludable, donde no imperaran los mecanismos posverdaderos, un líder político que empleara semejante alud de mentiras no podría contar con el apoyo de una gran parte de la ciudadanía. Sin embargo, lejos de ver minada la popularidad o la credibilidad de Donald Trump, hemos asistido a una pérdida de la confianza de la ciudadanía en estos tres medios de comunicación a los que observan cada vez con mayor suspicacia. La prueba palpable de que la ciudadanía estadounidense en general no ha dejado de mostrar su apoyo por el presidente Trump está en el propio resultado electoral de las elecciones de noviembre de 2020, en las que, si bien el presidente ha perdido las elecciones y deberá abandonar la Casa Blanca en enero, ha obtenido más de 74 millones de votos.

La revista Time le dedicó a esta cuestión una portada especialmente elocuente, la del 3 de abril de 2017, en la que aparecía el siguiente titular en letras rojas sobre fondo negro: «Is truth dead?» (Time, 2017). La expresión no es baladí. Asistimos en los últimos años a un uso inmoral de todo tipo de mentiras destinadas a la descripción del panorama político hasta un punto en el que parece que la verdad ya apenas tiene cabida. No solo porque le mentira se ha hecho con gran parte del campo político, sino también porque las afirmaciones verdaderas son puestas en 
cuestión e incluso elementos fuera de toda duda han sido disputados (ejemplos podrían ser los terraplanistas, los negacionistas del cambio climático o los movimientos antivacunas).

Cuando la distinción entre la verdad y la mentira va adquiriendo paulatinamente un carácter más y más difuso, una grave amenaza se cierne sobre el horizonte de una democracia. Sobre todo porque, dado el carácter del tiempo que nos ha tocado vivir, cada vez mayor número de ciudadanos elige, de una forma consciente o inconsciente, creer una afirmación apoyándose más en las apelaciones emotivas que esa afirmación suscita que en las referencias racionales y argumentos lógicos que se aporten. Uno de los primeros ejemplos demoledores del efecto de la posverdad se pudo constatar tras las afirmaciones de Kellyanne Conway, una de las asesoras de Donald Trump. La historia es rápida de contar. Tras la toma de posesión del cargo de presidente de Estados Unidos el día 20 de enero de 2017, algunas fuentes de la administración Trump dijeron que había ido mucha más gente a ese acto que a la toma de posesión de Barack Obama en 2009. Cuando las imágenes aéreas tomadas demostraron que esa afirmación era rotundamente falsa, Conway salió a la palestra para introducir un término demoledor: «nuestro jefe de prensa ha ofrecido otros datos alternativos» (D’Ancona, 2019: 24). Con la expresión «datos alternativos» o «hechos alternativos», como ocurrirá poco después, podemos constatar no solo que los mecanismos posverdaderos estaban poniéndose en marcha, sino más bien que ya estaban completamente asumidos. De este modo estaríamos dando pábulo a la idea de que no hay un concepto de verdad por el que pugnar, sino que todo queda reducido a la discusión entre unos hechos y otros hechos alternativos. La forma de contar la historia se torna más importante que los hechos que realmente ocurren. Sin embargo, nada de esto estaría ocurriendo 
si no fuera por el cuarto y último elemento de nuestro análisis, la carencia de pensamiento crítico en nuestro sistema educativo.

\section{Pensamiento crítico}

No es ningún secreto que la mayor parte de los sistemas educativos del mundo occidental están orientados hacia el desempeño profesional. La capacidad de introducirse en el mercado laboral y encontrar un empleo es lo que, aparentemente, persiguen nuestros modelos de educación. Esto no tendría que ser un rasgo negativo si no fuera porque está deteriorando algunos de los elementos esenciales para el sostenimiento de una democracia. En la adecuación del sistema educativo como un sistema de formación profesional se han ido perdiendo algunas parcelas que eran necesarias para el desarrollo de los futuros ciudadanos. No se trata solo de que las disciplinas humanísticas estén cada vez más capitidisminuidas en nuestras aulas, la cuestión no es solo que haya menos horas de filosofía, literatura, historia, música o artes, sino que, además, esas horas son sustituidas torpemente por algunas disciplinas instrumentales con un sesgo economicista, individualista y competitivo que no están contribuyendo a la mejora de las perspectivas sociales de nuestros jóvenes. De nada sirve eliminar la música a los estudiantes de 14 y 15 años y sustituirla por una asignatura de fomento de la actitud emprendedora. Si se pregunta a cualquier alto ejecutivo sobre su desempeño responderá fulminantemente que no habrá emprendimiento sin creatividad. Y si al mismo tiempo que tratamos de fomentar el emprendimiento erradicamos las asignaturas cuyos contenidos fomentan la creatividad estaríamos cayendo en un círculo vicioso cuya salida es obvia: la debacle educativa.

La introducción paulatina de los mecanismos de mercado en parcelas de las que antes estaban ausentes no es un asunto ba- 
ladí. El mercado tiene un efecto corrosivo demoledor cuando se entromete donde no le debería corresponder. La educación es una de estas parcelas. Los mecanismos de mercado terminan provocando dos problemas: primero fomentan la injusticia y segundo la corrupción. El filósofo norteamericano Michael Sandel así lo ha puesto de manifiesto con multitud de ejemplos en su libro Lo que el dinero no puede comprar (Sandel, 2019). Platón nos puede ayudar convenientemente en este momento. En la República, Platón dice que la primera obligación del estado es cubrir las necesidades básicas de sus ciudadanos. Sin embargo, el discípulo más famoso de Sócrates inmediatamente continúa diciendo que con esto no estaríamos sino ocupándonos meramente de las necesidades animales de los seres humanos. Estas necesidades animales ocuparían un lugar mucho menos relevante que las verdaderas necesidades importantes de los ciudadanos: la paideia. Lo que dice Platón es que inmediatamente después de cubrir las necesidades básicas de los ciudadanos el estado lo que tiene que hacer es ocuparse de que la paideia se fomente adecuadamente entre todos los habitantes de la polis. La palabra paideia es un concepto complejo y de enorme calado en la cultura griega. Para nuestro tiempo se podría traducir algo así como las ideas de educación y cultura en una sola palabra. Así pues, para Platón el fomento de la educación y de la cultura es el elemento esencial del que se tiene que ocupar el estado que quiera mantener una polis equilibrada (Platón, 2003). Si partimos de esta premisa, podemos constatar que el fomento de la paideia en nuestro tiempo se ha devaluado, por cuanto la meta casi exclusiva que se persigue en el ámbito educativo es una meta profesional, olvidando en el camino la necesidad de desarrollar una serie importante de capacidades vinculadas con el pensamiento crítico, el razonamiento, la argumentación y la lógica que son cada día más necesarias. Esto es así, sobre todo, a la luz de los nuevos tiempos y todos 
los mecanismos que hemos puesto de manifiesto en las páginas anteriores. Es a causa de la clamorosa ausencia de los contenidos de pensamiento crítico por lo que un porcentaje elevado de la población está dispuesto a pasar por las Horcas Caudinas de la más clamorosa credulidad frente a afirmaciones rotundamente falsas. Entre los elementos básicos del pensamiento crítico se enseña a distinguir entre las fuentes de los datos que se aporten, a desgranar entre un hecho, una afirmación y una mera opinión, etc. (Ruiz, 2019).

Los resultados del sistema educativo no se pueden medir exclusivamente con las pruebas del informe PISA. El informe PISA es un conocido instrumento diseñado desde una óptica ideológica neoliberal (Fernández González, 2015; Fernández, García y Galindo, 2017; Saura y Geo, 2016). Tiene cierta utilidad porque permite comparar diversos modelos de educación entre diferentes países, sin embargo, su principal debilidad radica en el sesgo economicista del que parte. Las pruebas son evidentes. Cuando una población carece de las herramientas básicas de pensamiento crítico para defenderse frente al discurso sesgado, parcial y tendencioso tan frecuente de nuestro tiempo, el resultado es un aumento de la fragilidad de la democracia. Como hemos recordado en páginas anteriores, el verdadero enemigo de la democracia es la demagogia. Si esta además halla el terreno abonado para extender sus semillas espurias todo estará perdido a medio y largo plazo. Los datos son elocuentes. Según un informe de la Unesco del año 2019, uno de cada tres estudiantes de secundaria (un 32\%) ha sufrido algún tipo de acoso o intimidación «por sus compañeros en la escuela al menos una vez en el último mes y una proporción similar se ha visto afectada por violencia física» (Unesco, 2019). Este tipo de violencia presente en las aulas demuestra el fracaso del modelo educativo a la hora 
de fomentar esa educación y cultura de la que hablaba Platón. La ausencia de paideia hace más frágiles y vulnerables a los jóvenes estudiantes. Existe evidencia empírica que sugiere que a una mayor presencia de pensamiento crítico entre los estudiantes de secundaria se corresponde una mayor resistencia frente al acoso y la intimidación (Hamodi Galán y Jiménez Robles, 2018; Ortega et al., 2005). Luego el dato de la macroencuesta de la Unesco demuestra la carencia de contenidos de pensamiento en la inmensa mayoría de los países del mundo entero.

«La primera operación mental es distinguir lo verdadero de lo falso» Albert Camus, Sísifo

\section{Conclusión}

Estos cuatro elementos que hemos descrito someramente (la demagogia y el populismo, las noticias falsas, la posverdad y la carencia de pensamiento crítico) son un auténtico contubernio amenazador para cualquier democracia. La fragilidad de nuestro tejido social de cara a afrontar el desafío que se nos presenta de manos de estos elementos es evidente. Una democracia que no penaliza el uso de la mentira como herramienta política es una democracia corrupta, moribunda. Lamentablemente, la historia de la humanidad no es un péndulo y no podemos afirmar que estos mecanismos corrosivos vayan a retroceder por sí solos. La línea que separa la civilización de la barbarie pasa, hoy en día, por defender la idea de la verdad, de la honestidad comunicativa. Bajar los brazos frente a esta tarea inmensa supondrá una pérdida irreparable. Decía Wittgenstein en la proposición 5.6 de su Tractatus que los límites de mi lenguaje son los límites de mi 
mundo. Por otra parte, se ha demostrado empíricamente en un artículo muy influyente que el discurso político contemporáneo es el más pedestre y simplificador que ha existido desde que se guarda memoria de las intervenciones públicas de los políticos (Jordan et al., 2019). Dicho con otras palabras, los políticos se dirigen voluntariamente a su auditorio como si este estuviera compuesto por niños de entre 9 y 11 años. Esto podría parecer un afán de ganar en claridad y comprensión. Sin embargo, nuestra lectura es mucho más siniestra: conviene recordar que George Orwell, con una terrible capacidad de anticipación, escribió en su novela 1984 el afán principal del régimen totalitario por imponer la neolengua no era otro que el de reducir la capacidad de reflexión de los ciudadanos y facilitar el dominio y el control sobre ellos. Si los límites de mi lenguaje son los límites de mi mundo, cuanto más estrecho sea mi lenguaje más miope será mi mundo. Será más fácilmente controlable. Y los límites entre la verdad y la mentira habrán desaparecido. 


\section{REFERENCIAS BIBLIOGRÁFICAS}

Aristóteles (1988). Política. Madrid: Gredos.

- (1994). Retórica. Madrid: Gredos.

Barthes, Roland (1999). «El mundo de la lucha libre». En Mitologías. Madrid: Siglo XXI, pp. 8-14.

Bermúdez Vázquez, Manuel y Casares Landauro, Elena (2019). «El problema de la posverdad en la sociedad contemporánea». En Bermúdez, Manuel y Ballesteros, Lucía (eds.). Comunicación digital: retos y oportunidades. Sevilla: Egregius, pp. 55-68.

Bermúdez VÁzQuez, Manuel (2019). «Análisis del concepto 'posverdad' desde la óptica de la retórica clásica». Diálogo filosófico, 105, pp. 341-352.

Comisión Europea (2019). «Unión Europea vs Desinformación». 5 de noviembre. Disponible en: https:/ / ec.europa.eu/ spain/news/20191105_eu-vs-disinformation_es [Consulta: 7 noviembre 2020].

(2020). «Lucha contra la desinformación». Agosto de 2020. Disponible en: https://ec.europa.eu/info/live-worktravel-eu/coronavirus-response/fighting-disinformation_es [Consulta: 7 noviembre 2020].

D’Ancona, Matthew (2019). Posverdad. La nueva guerra contra la verdad y cómo combatirla. Madrid: Alianza.

RAE (2020). «Posverdad». Disponible en: https://dle.rae.es/ posverdad? $\mathrm{m}=$ form [Consulta: 7 diciembre 2020].

Eurostat (2020). «Estadísticas sobre sociedad y economía digital-Hogares y particulares». 19 de octubre. Disponible en: https://ec.europa.eu/eurostat/statistics-explained/index. php?title=Digital_economy_and_society_statistics_-_households_and_individuals/es [Consulta: 7 diciembre 2020]. 
Fernández GonzÁlez, Noelia (2015). «Pisa como instrumento de legitimación de la reforma de la LOMCE». Sociedad Española de Pedagogía, 67(1), pp. 165-178.

Fernández Liria, Carlos, García Fernández, Olga y Galindo FERrández, Enrique (2017). Escuela o barbarie. Entre el neoliberalismo salvaje y el delirio de la irquierda. Madrid: Akal.

Fundéu (2016). «Populismo, palabra del año 2016 para la Fundéu». 30 de diciembre. Disponible en: https://www.fundeu. es/recomendacion/populismo-palabra-del-ano-2016-para-la-fundeu-bbva [Consulta: 27 octubre 2020].

- (2017). «Ciberanzuelo, la alternativa favorita de nuestros seguidores a clickbait». 25 de abril. Disponible en: https:// www.fundeu.es/noticia/ciberanzuelo-la-alternativa-favorita-de-nuestros-seguidores-a-clickbait/ [Consulta: 7 diciembre 2020].

Gartner (2017). «Gartner Reveals Top Predictions for IT Organizations and Users in 2018 and Beyond». 3 de octubre. Disponible en: https://www.gartner.com/en/newsroom/ press-releases /2017-10-03-gartner-reveals-top-predictionsfor-it-organizations-and-users-in-2018-and-beyond [Consulta: 7 diciembre 2020].

Hamodi Galán, Carolina y Jiménez Robles, Leire (2018). «Modelos de prevención del bullying: ¿qué se puede hacer en educación infantil? Revista de Investigación Educativa de la REDIECH, vol. 9, núm. 16. Disponible en: http://www.scielo.org.mx/scielo.php?scrip$\mathrm{t}=$ sci_arttext\&pid=S2448-85502018000100029\#B24 [Consulta: 21 diciembre 2020].

Jordan, Kayla, Sterling, Joanna, Pennebaker, James y Boyd, Ryan (2019). «Examining long-term trends in politics and culture through language of political leaders and cultural institutions». Proceedings of the National Academy of Sciences, 116, pp. 3476-3481. 
Leith, Sam (2011). Are you talking to me: Rhetoric from Aristotle to Obama. Londres: Profile Books.

Martínez Pradales, David (2019). «En 2022 consumiremos más noticias falsas que verdaderas». 2 de abril. Disponible en: https://www.nobbot.com/general/noticias-falsas-2022 [Consulta: 7 diciembre 2020].

McIntyre, Lee (2020). Posverdad. Madrid: Cátedra.

Nicolás, Juan Antonio (2019). «Posverdad: cartografía de un fenómeno complejo». Diálogo Filosófico, 105, pp. 302-340.

Ortega, Sylvia, Ramírez, Marco y Castelán, Adrián (2005). «Estrategias para prevenir y atender el maltrato, la violencia y las adicciones en las escuelas públicas de la Ciudad de México». Revista Iberoamericana de Educación, 38, pp. 147-169.

Oxford Languages (2016). «Word of the year 2016», Disponible en: https:/ / languages.oup.com/word-of-the-year/2016 [Consulta: 27 octubre 2020].

Platón (2003). República. Madrid: Gredos.

Rodríguez, Roberto (2008). «La tradición liberal». En Quesada, Fernando (ed.). Ciudady ciudadanía. Senderos contemporáneos de la filosofía política. Madrid: Trotta, pp. 7-30.

Ruiz, José Carlos (2019). El arte de pensar. Córdoba: Berenice.

SANDEL, Michael (2019). Lo que el dinero no puede comprar. Madrid: Debate.

Saura, Geo y Muñoz Moreno, José Luis (2016). «Prácticas neoliberales de endo-privatización y nuevas formas de resistencia colectiva en el contexto de la política educativa española». Revista Educación, Politica y Sociedad, 1(2), pp. 43-72.

Shearer, Elisa y Matsa, Katerina Eva (2018). «News Use Across Social Media Platforms 2018». 10 de septiembre 2018. Disponible en: https://www.journalism.org/2018/09/10/ news-use-across-social-media-platforms-2018. [Consulta: 7 diciembre 2020]. 
TARín SAnz, Adrián (2018). «Comunicación, ideología y poder: Anotaciones para el debate entre la Teoría de la Propaganda Intencional y la Teoría de la Reproducción Espontánea de la Propaganda». Comunicación y sociedad, 32, pp. 191-209.

The Guardian (2020). «Tsunami of untruths': Trump has made 20.000 false or misleading claims-report». 13 de julio. Disponible en: https://www.theguardian.com/us-news/2020/ jul/13/donald-trump-20000-false-or-misleading-claims [Consulta: 7 diciembre 2020].

Time (2017). «Is truth death?». 3 de abril. Disponible en: https://time.com/magazine/us/4710599/april-3rd-2017-vol189-no-12-u-s [Consulta: 20 diciembre 2020].

UnESCO (2019). «Behind the numbers: Ending school violence and bullying». 22 de enero. Disponible en: https:// es.unesco. org/news/violencia-y-acoso-escolares-son-problema-mundial-segun-nuevo-informe-unesco [Consulta: 21 diciembre 2020].

Washington Post (2020). «President Trump has made more than 20.000 false or misleading claims». 13 de julio. Disponible en: https://www.washingtonpost.com/ gdpr-consent/?next_url=https $\% 3 \mathrm{a} \% 2 \mathrm{f} \% 2 \mathrm{fwww}$.washingtonpost.com $\% 2$ fpolitics $\% 2$ f2020\%2f07\%2f13\%2fpresident-trump-has-made-more-than-20000-false-or-misleading-claims $\% 2 f$ [Consulta: 7 diciembre 2020]. 